\title{
Lesions of the paraventricular nucleus of the thalamus differentially affect sign- and goal-tracking conditioned responses
}

\author{
Joshua L. Haight, ${ }^{1}$ Kurt M. Fraser, ${ }^{2}$ Huda Akil ${ }^{3,4}$ and Shelly B. Flagel ${ }^{1,3,4}$ \\ ${ }^{1}$ Neuroscience Graduate Program, University of Michigan, Ann Arbor, MI, USA \\ ${ }^{2}$ Undergraduate Program in Neuroscience, University of Michigan, Ann Arbor, MI, USA \\ ${ }^{3}$ Department of Psychiatry, University of Michigan, Ann Arbor, MI, USA \\ ${ }^{4}$ Molecular and Behavioral Neuroscience Institute, University of Michigan, Ann Arbor, MI 48109-5720, USA
}

Keywords: incentive salience, motivation, paraventricular nucleus of the thalamus, Pavlovian conditioning, rats

Edited by Jeffrey Dalley

Received 22 May 2015, accepted 27 July 2015

\begin{abstract}
Recently, evidence has emerged suggesting a role for the paraventricular nucleus of the thalamus (PVT) in the processing of reward-associated cues. However, the specific role of the PVT in these processes has yet to be elucidated. Here we use an animal model that captures individual variation in response to discrete reward-associated cues to further assess the role of the PVT in stimulus-reward learning. When rats are exposed to a Pavlovian conditioning paradigm, wherein a discrete cue predicts food reward, two distinct conditioned responses emerge. Some rats, termed sign-trackers, approach and manipulate the cue, whereas others, termed goal-trackers, approach the location of reward delivery upon cue presentation. For both sign- and goal-trackers the cue is a predictor, but only for sign-trackers is it also an incentive stimulus. We investigated the role of the PVT in the acquisition and expression of these conditioned responses using an excitotoxic lesion. Results indicate that PVT lesions prior to acquisition amplify the differences between phenotypes - increasing sign-tracking and attenuating goal-tracking behavior. Lesions of the PVT after rats had acquired their respective conditioned responses also attenuated the expression of the goal-tracking response, and increased the sign-tracking response, but did so selectively in goal-trackers. These results suggest that the PVT acts to suppress the attribution of incentive salience to reward cues, as disruption of the functional activity within this structure enhances the tendency to sign-track.
\end{abstract}

\section{Introduction}

It is well established that environmental stimuli that are repeatedly paired with rewards can acquire motivational control over behavior, and do so through complex cortico-striatal-thalamic brain networks (Pierce \& Kalivas, 1997; Kelley et al., 2005). Within the past 10 years, evidence has emerged suggesting that the paraventricular nucleus of the thalamus (PVT) is a critical component of this circuitry (Martin-Fardon \& Boutrel, 2012; James \& Dayas, 2013), and its anatomical location supports a role for modulating cue-motivated behaviors (Vertes \& Hoover, 2008; Li \& Kirouac, 2012). In agreement with this, it has been shown that discrete cues associated with both natural rewards and drugs of abuse elicit robust activity in the PVT. For example, repeated pairings of a cue light with a water reward in a Pavlovian manner elicit enhanced c-fos expression in the

Correspondence: Dr Shelly B. Flagel, ${ }^{4}$ Molecular and Behavioral Neuroscience Institute, as above.

E-mail: sflagel@umich.edu
PVT relative to controls exposed to random cue-reward presentations (Igelstrom et al., 2010). In addition, elevated c-fos levels are found in the PVT following cue-induced reinstatement of drug-seeking behaviors (Wedzony et al., 2003; Dayas et al., 2008; James et al., 2011). Taken together, these data suggest that the PVT may be involved in mediating cue-motivated behaviors, including Pavlovian conditioned responses (CRs), but its specific role in these processes is unknown.

Importantly, Pavlovian conditioned reward cues can act as both predictive and incentive stimuli, and individuals differ in the extent to which they attribute reward cues with motivational properties (Flagel et al., 2009; Robinson \& Flagel, 2009). When rats are exposed to a Pavlovian conditioned approach (PCA) paradigm, wherein a discrete cue (conditioned stimulus; CS) predicts a food reward (unconditioned stimulus), some rats, termed sign-trackers (STs), will develop a CR directed towards the cue. For these individuals, the cue itself becomes attractive, eliciting approach, and desired, such that STs will work to obtain it in the absence of a food reward (Robinson \& Flagel, 2009). For others, termed goal-trackers (GTs), the cue elicits a CR directed towards the site of reward delivery. Thus, the cue is a predictive stimulus for both STs and GTs, and is effective at evoking a CR in both 
groups of animals, but only for the STs is the cue imbued with incentive salience, rendering it attractive and desired (Robinson \& Flagel, 2009).

Using the ST/GT animal model, it has been shown that presentation of an incentive stimulus previously paired with a food or drug reward can elicit robust c-fos expression in the PVT (Flagel et al., 2011a; Yager et al., 2014). In addition, STs and GTs show different patterns of 'functional connectivity' (correlated levels of c-fos mRNA) between the PVT and other brain areas following cue presentation, suggesting the PVT might differentially regulate these CRs (Flagel et al., 2011a; Haight \& Flagel, 2014). While these data further support the notion that the PVT is involved in cue-motivated behaviors, a causal link between the PVT and PCA behavior has yet to be established. In addition, it is unknown whether the PVT is necessary for the acquisition of PCA behavior, or whether it is also critical for the ongoing expression of Pavlovian CRs after they have been acquired. Here we used excitotoxic lesions to specifically

\section{A Surgery}
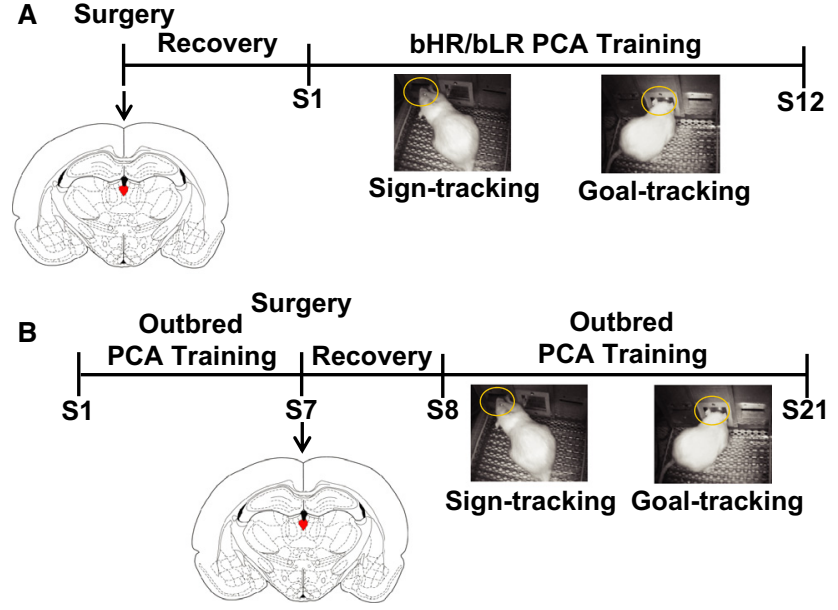

FIG. 1. Timeline for (A) Experiment 1 (Acquisition) and (B) Experiment 2 (Expression) with representative photos capturing sign- and goal-tracking conditioned responses. bHR, selectively bred high-responder; bLR, selectively bred low-responder; PCA, Pavlovian conditioned approach. investigate the role of the PVT in the acquisition and expression of sign- and goal-tracking CRs. Based on our previous findings (Flagel et al., 2011a; Haight \& Flagel, 2014; Yager et al., 2014), we hypothesized that the PVT is an integral part of the neural circuitry underlying the attribution of incentive salience to reward cues, and that lesions of the PVT would disrupt both the acquisition and the expression of sign- and goal-tracking behaviors.

\section{Materials and methods}

All experiments followed the Guide for the Care and Use of Laboratory Animals: Eighth Edition, revised in 2011, published by the National Academy of Sciences, and all procedures were approved by the University of Michigan University Committee for the Use and Care of Animals.

\section{Experiment 1: The effects of PVT lesions on the acquisition of sign- and goal-tracking CRs}

\section{Subjects}

Subjects were 52 adult male Sprague-Dawley rats from generations F38 and F40 of selectively bred high-responder (bHR) and low-responder (bLR) rat lines (Stead et al., 2006). These rats have been bred based on their locomotor response to a novel environment. bHR rats show increased locomotor response to novelty relative to bLRs, who show relatively low levels of activity in a novel environment. Importantly, several other traits seem to have been co-selected in these rat lines, including sign- and goal-tracking. It has previously been shown that bHR rats are primarily STs, and bLRs are GTs (Flagel et al., 2010, 2011b, 2014). That is, we can predict with 90$100 \%$ certainty whether these rats will develop a sign-tracking or a goal-tracking CR based on their breeding history. Knowing this information a priori therefore allows us to examine the effects of experimental manipulations on the acquisition of these CRs.

Rats were drawn from 7 to 11 litters per phenotype within each generation. No more than four pups from any given litter were used

\section{A}

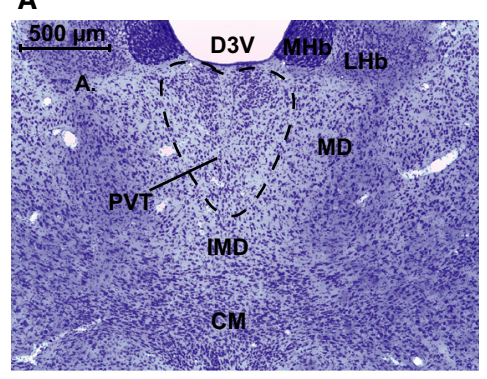

B

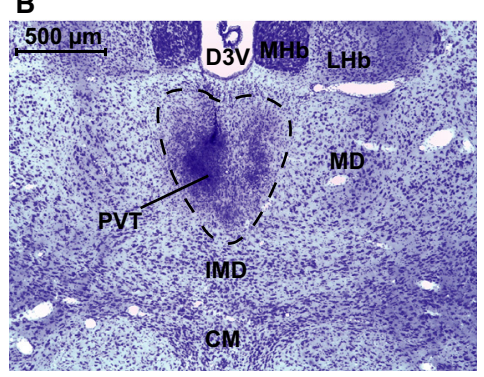

C
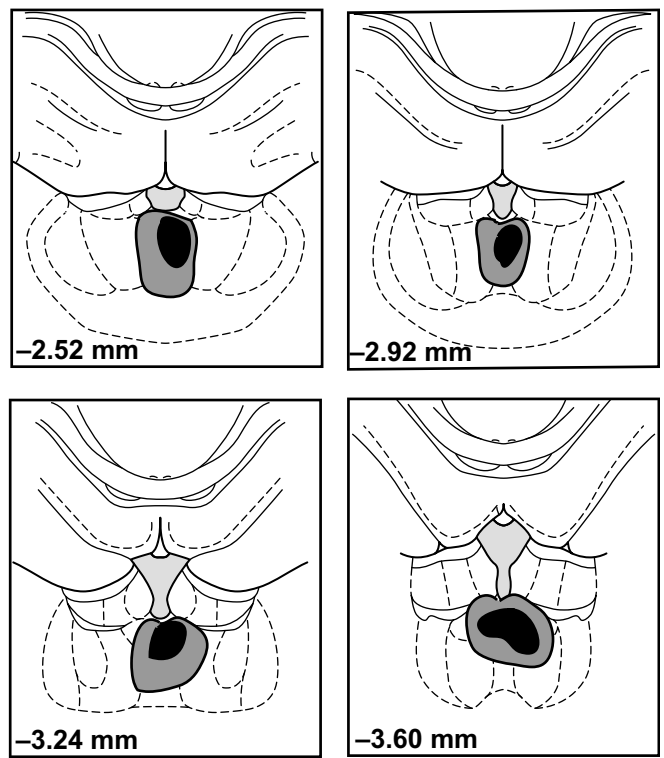

FIG. 2. Histological analysis of lesion sites for Experiment 1. Photomicrograph showing representative cresyl-violet stained sections of (A) an intact PVT and (B) a PVT lesion; approximate bregma level $=\mathrm{AP}-3.00$; scale bar $=500 \mu \mathrm{m}$. (C) Illustration showing the approximate largest (gray) and smallest (black) accepted excitotoxic lesions of the PVT for Experiment 1. 
and littermates were balanced across treatment groups within a phenotype. Thus, a maximum of two rats from the same litter were assigned to a single treatment group. Rats were approximately 65 days of age at the start of the study. Prior to surgery, rats were pair-housed with the same phenotype in acrylic cages $(46 \times 24 \times 22 \mathrm{~cm})$ in a temperature-controlled room and maintained on a 12-12-h light-dark cycle, with lights on at 06:00 h. Food and water were available ad libitum. Following surgery, all rats were single housed under the same conditions.

\section{Surgery}

Prior to behavioral training, all subjects underwent lesion or sham surgery (for Experimental Timeline, see Fig. 1A). All surgery was performed under aseptic conditions. Rats were anesthetized with isoflurane and placed in a stereotaxic device. The scalp was shaved, sanitized with $70 \%$ alcohol followed by Betadine (Stamford, CT, USA) solution and incised to expose the cranium. The skull was leveled, and small holes were drilled above the PVT. A 33-gauge injector (PlasticsOne, Roanoke, VA, USA), connected to a $1-\mu \mathrm{L}$ Hamilton syringe (Hamilton Company, Reno, NV, USA) via P50 tubing, was then lowered into two sites in the PVT at the following coordinates measured from bregma (based on Hamlin et al., 2009): A-P -2.6, M-L 0.2, D-V -5.5; and AP -3.6, M-L 0.3, D-V -5.6. To produce a lesion, $200 \mathrm{~nL}$ of $0.06 \mathrm{~m}$ ibotenic acid (Abcam, Cambridge, MA, USA) dissolved in sterile-filtered $0.1 \mathrm{~m}$ phosphatebuffered saline $(\mathrm{pH} 7.3-7.4)$ was injected at a rate of $100 \mathrm{~nL} / \mathrm{min}$ using a syringe pump (Harvard Apparatus, Holliston, MA, USA). Control rats received infusions of vehicle (phosphate-buffered saline) only. Following infusion, the injector was left in place for $5 \mathrm{~min}$ to minimize diffusion up the injection track upon removal. Immediately prior to surgery, as well as $24 \mathrm{~h}$ later, rats received subcutaneous injections of $2.5 \mathrm{mg} / \mathrm{kg}$ flunixin for pain management. Rats were allowed to recover in their home cages for 5-7 days prior to any behavioral testing.

\section{Pavlovian conditioning procedures}

The equipment and procedures used for PCA training have been described previously in detail (Flagel et al., 2007, 2008). Sixteen standard test chambers (MED Associates, St. Albans, VT, USA) were used. Each chamber was equipped with a pellet dispenser and food cup located in the middle of the front wall. An illuminated, retractable lever was located to either the left or the right (counterbalanced) of the food cup at equal height. All levers were set so that $10 \mathrm{~g}$ of force caused a deflection of the lever and would result in a 'lever contact' being recorded. A white house light was located at the top of the wall opposite the food cup and lever, and was illuminated for the duration of the training sessions. Operation of the pellet dispenser resulted in the delivery of one 45-mg banana-flavored food pellet (Bio-Serv, Flemington, NJ, USA) into the food cup. Head entries into the food cup were detected by an infrared photo beam. Each chamber was housed in a sound-attenuated box equipped with a ventilation fan that generated background noise.

All training was conducted between 13:00 and 17:00 h. For 2 days prior to training, rats were briefly handled by the experimenter, and banana-flavored food pellets (approximately 25-30 pellets per rat) were delivered into the rats' home cages to familiarize them with the reward to be used in training. Two pre-training sessions were then conducted prior to Pavlovian conditioning. During these sessions the house light was illuminated and 50 food pellets were delivered one at a time into the food cup on a variable interval 30-s schedule, and the lever remained retracted for the duration of the session. Each pre-training session lasted approximately $25 \mathrm{~min}$, and by the end of the second session rats were reliably retrieving the majority of their food pellets. Following pre-training, rats underwent 12 Pavlovian conditioning sessions, one session per day. Rats were trained for seven consecutive days, given a 2-day break and then trained for five more consecutive days, for a total of 12 sessions. Each training session consisted of 25 trials in which an illuminated lever (CS) was inserted into the test chamber for $8 \mathrm{~s}$, and then immediately upon its retraction a food pellet (unconditioned stimulus) was delivered into the food cup. The lever-CS was presented on a variable interval 90-s schedule (range 30-150 s), and the session lasted approximately $40 \mathrm{~min}$.

The following events were recorded by MED Associates software: (1) the number of lever contacts, (2) the latency to first lever contact, (3) the number of head entries into the food cup during the 8-s lever presentation, (4) the latency to first food cup entry upon lever presentation and (5) the number of head entries into the food cup during the inter-trial interval (ITI). These measures allowed us to quantify sign-tracking (lever-CS directed) and goal-tracking (foodcup directed) behavior, as well as activity during the ITI.

\section{Experiment 2: The effects of PVT lesions on the expression of sign- and goal-tracking CRs}

\section{Subjects}

Subjects were 120 adult male Sprague-Dawley rats obtained from two commercial vendors (Charles River Laboratories, Portage, MI, USA; Harlan Laboratories, Indianapolis, IN and Haslett, MI, USA). Rats were ordered from two different vendors to get an adequate number of sign- and goal-trackers (Fitzpatrick et al., 2013). Rats were approximately 60 days of age at the time of arrival and allowed to acclimate for 10-14 days prior to any handling or behavioral testing. Rats were pair housed in acrylic cages $(46 \times 24 \times 22 \mathrm{~cm})$ in a temperature-controlled room and maintained on a 12-12-h light-dark cycle, with lights on at 06:00 h. Food and water were available ad libitum. All training was conducted between 13:00 and 17:00 h.

\section{Pavlovian conditioning procedures}

Following the 10-14-day acclimation period, rats were handled and given banana pellets in their home cages for 2 days. Rats then underwent two pre-training sessions identical to those described in Experiment 1 , to ensure rats were reliably consuming their food pellets. Following pre-training, rats underwent seven sessions of Pavlovian conditioning (as described above; for an experimental timeline, see Fig. 1B). Importantly, unlike Experiment 1, this training occurred before surgery. Surgery was performed after seven Pavlovian training sessions because previous studies have indicated that rats acquire the CRs and approach asymptotic performance within the first week of training (Robinson \& Flagel, 2009; Flagel et al., 2011a).

Rats were characterized as STs, GTs or intermediate responders based on the average PCA Index scores (Meyer et al., 2012) from sessions 6 and 7 of training. Briefly, the PCA Index score is a composite measure used to quantify the degree to which an individual's behavior is directed towards the lever-CS or the food cup. The PCA Index score is based on three measures of Pavlovian approach behavior: the response bias for contacting the lever or food cup [(total lever-directed contacts - total food cup-directed contacts) $\div$ (sum of total contacts)], the probability of lever or food cup contact 
$\left[\operatorname{Prob}_{(\text {lever })}-\operatorname{Prob}_{(\mathrm{Mag})}\right]$, and the latency to contact the lever or enter the food cup [(food cup entry latency - lever contact latency) $\div 8$ ] These three values are then used to calculate the PCA Index score: [(response bias score + probability difference score + latency difference score) $\div 3$ ]. PCA Index scores range from +1.0 to -1.0 , with +1.0 representing a rat whose behavior is exclusively directed towards the lever, and -1.0 representing a rat whose behavior is exclusively directed towards the food cup. In the current study, rats that were classified as STs had PCA Index scores ranging from 0.5 to 1.0 , while rats classified at GTs had scores ranging from -0.5 to -1.0 (Meyer et al., 2012).

Sign- and goal-trackers were then assigned to PVT lesion or sham treatment groups, which were balanced per phenotype based on both PCA Index scores and Vendor (Harlan vs. Charles River). Importantly, although there were more STs from the Harlan population and more GTs from Charles River (as found in Fitzpatrick et al., 2013), there were no behavioral differences based on Vendor within each phenotype. The average PCA Index scores pre-lesion were similar across Vendor groups (ST, Charles River $=0.80$; ST, Harlan $=0.84$; GT, Charles River $=-0.72$; GT, Harlan $=-0.68$, such that behavior exhibited by STs from Harlan was indistinguishable from Charles River STs, and the same was true when comparing GTs from each vendor.

\section{Surgery}

The procedures for surgery were identical to those used in Experiment 1. However, for this experiment, surgical coordinates were altered to lesion a greater extent of the PVT. Ibotenic acid or vehicle was injected at the following coordinates from bregma, with the stereotaxic arm at a $10^{\circ}$ angle toward the midline: A-P -2.0 , M-L 1.0, D-V -5.4; A-P -3.0, M-L 1.0, D-V -5.5. Following a 5-7day recovery period from surgery, rats underwent 14 additional sessions of PCA training to assess the effects of PVT lesions on the performance of sign- and goal-tracking CRs.

\section{Histological analysis of lesion sites (Experiments 1 and 2)}

Following the completion of each experiment, rats were deeply anesthetized with a mixture of ketamine and xylazine $(90 \mathrm{mg} / \mathrm{kg}$ ketamine; $10 \mathrm{mg} / \mathrm{kg}$ xylazine) and transcardially perfused with approximately $100 \mathrm{~mL}$ of $0.9 \%$ saline, followed by $200 \mathrm{~mL}$ of $4 \%$ paraformaldehyde in $0.1 \mathrm{M}$ phosphate-buffered saline ( $\mathrm{pH} 7.4$ ). Brains were extracted from the skull and post-fixed overnight in $4 \%$ paraformaldehyde at $4{ }^{\circ} \mathrm{C}$. Brains were then cryoprotected in graduated sucrose solutions $(10-30 \%$ in $0.1 \mathrm{M}$ sodium phosphate buffer, $\mathrm{pH}$ 7.4) at $4{ }^{\circ} \mathrm{C}$ over 3 days. Following cryoprotection, brains were mounted in Tissue-Plus O.C.T. compound (Thermo Fisher Scientific, Hampton, NH, USA), frozen and coronally sectioned on a cryostat at a thickness of $40 \mu \mathrm{m}$. Sections were mounted onto SuperFrost Plus slides (Thermo Fisher Scientific), stained with cresyl violet, dehydrated in graduated ethanol solutions followed by two xylenes washes, and coverslipped with Permount coverslipping medium (Thermo Fisher Scientific). To determine the presence of a PVT lesion, histological analysis was performed by an experimenter blind to treatment conditions. Lesions were identified by gliosis and a lack of cell bodies in the area of interest (Fig. 2A and B).

\section{Statistical methods (Experiments 1 and 2)}

All statistical analyses were performed with the SPSS Statistics program, version 21 (IBM, Armonk, NY, USA). Changes in PCA behavior across sessions, measured by contacts, latency to contact and probability of contact for either the lever or the food cup, were evaluated using linear mixed-effects models (Verbeke \& Molenberghs, 2000), in which Session, Phenotype (bHR/bLR) and Treatment (lesion vs. sham) were treated as independent variables. The covariance structure was explored and modeled appropriately for each dependent variable. A repeated-measure ANOvA was used to further assess the difference in sign- and goal-tracking behaviors pre- vs. post-lesion, with Treatment and Block (pre-lesion vs. postlesion) as independent variables. For all analyses significance was set at $P \leq 0.05$ and Bonferroni post-hoc analyses were used to correct for multiple comparisons when significant main effects or interactions were found.

\section{Results}

Experiment 1: The effects of PVT lesions on the acquisition of sign- and goal-tracking CRs

\section{Histology}

Figure $2 \mathrm{C}$ shows a schematic representation of the lesion size and location for rats included in the study. In general, lesions spanned -2.3 to $-3.8 \mathrm{~mm}$ posterior to bregma, encompassed the entire PVT and only minimally damaged surrounding thalamic nuclei. Rats with small lesions that did not encompass the borders of the PVT, or lesions that resulted in extensive damage to neighboring nuclei or the hippocampus, were eliminated from the data analyses. Based on these criteria, nine rats were excluded from the study and the following were included: bHR Lesion $n=9$, bHR Sham $n=12$, bLR Lesion $n=12$, bLR Sham $n=10$.

\section{Effects of PVT lesions in bLR rats}

As indicated in Table S1, there was a significant Phenotype $\times$ Session and/or Phenotype $\times$ Session $\times$ Treatment interaction for all measures of sign- and goal-tracking behaviors in Experiment 1 . Thus, independent analyses were conducted for bLRs and bHRs, as described below.

For bLR rats, lesions of the PVT prior to acquisition of the CR affected goal-tracking (Fig. 3A-C), but not sign-tracking behaviors (Fig. S1D-F). Linear mixed-effects models revealed a significant effect of Treatment $\left(F_{11,20}=4.48, \quad P=0.01\right)$ and a Session $\times$ Treatment interaction $\left(F_{11,20}=2.33, P=0.05\right)$ for food cup contacts (Fig. 3A). There was a significant within-group effect of Session in bLR Sham rats $\left(F_{11,20}=4.47, P=0.002\right)$, but not bLR Lesion rats, demonstrating that only bLR Sham rats learned a goaltracking CR over the course of training. In support of this, there was a trend towards significance for an effect of Treatment on measures of probability of food cup entry $\left(F_{11,20}=3.77, P=0.07\right.$; Fig. 3B) and latency to food cup entry $\left(F_{11,20}=4.00, P=0.06\right.$; Fig. $3 C$ ), but these effects did not reach statistical significance.

To determine whether PVT lesions were affecting general levels of activity in bLR rats when the CS was not present, we examined food cup responding during the inter-trial interval. While there was no significant effect of Treatment on this measure, there was a significant Session $\times$ Treatment interaction $\left(F_{11,20}=3.49, P=0.01\right.$; Fig. S2). However, further analyses revealed a significant difference between bLR Sham and bLR Lesion groups only on Session $8\left(F_{1,20}=7.22, P=0.01\right)$, which happened to be the first session of training after a 2-day break. Thus, although bLR Lesion rats had a tendency to enter the food cup with less frequency than 


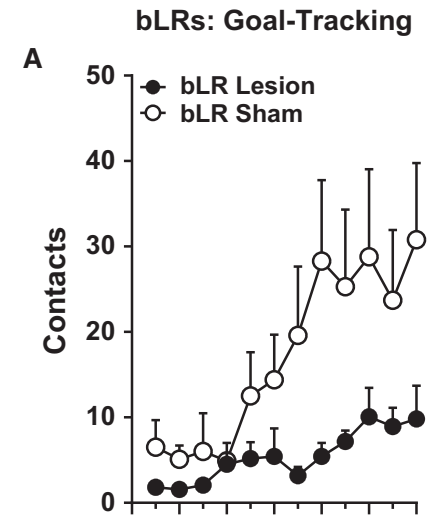

B

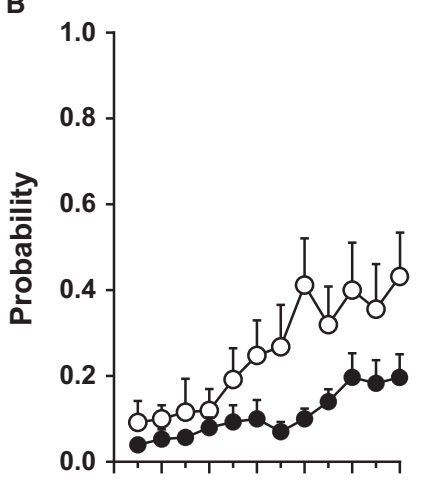

C

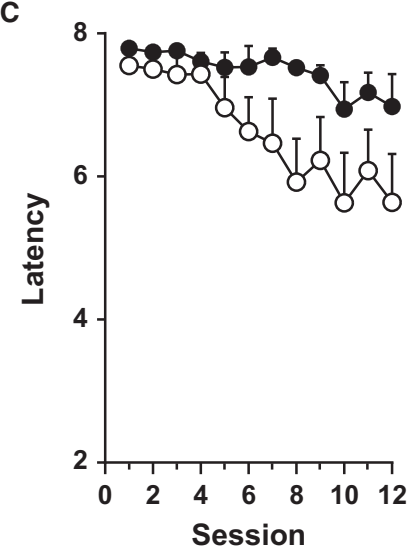

bHRs: Sign-Tracking

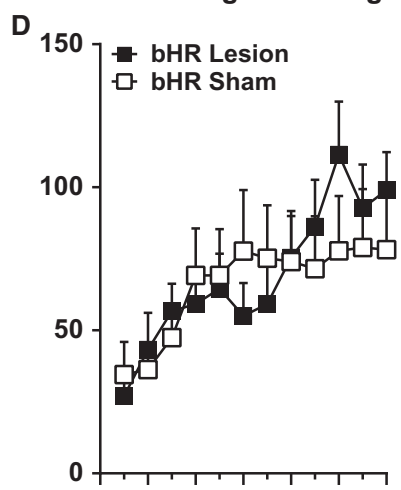

E

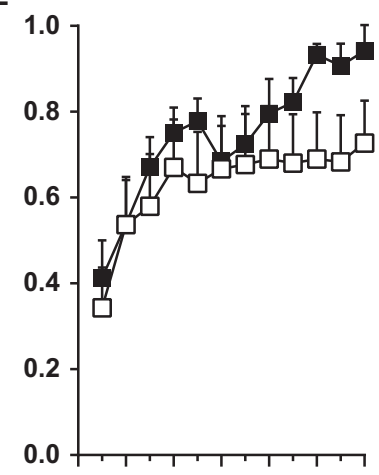

$\mathbf{F}$

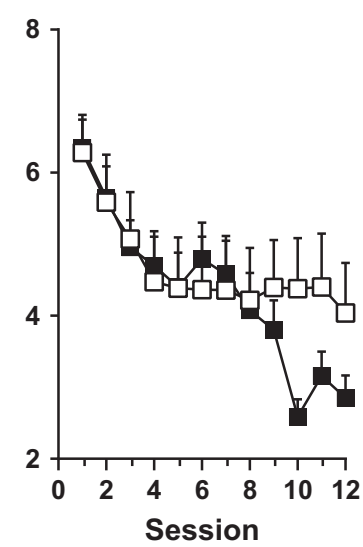

FIG. 3. Effects of PVT lesion on the acquisition of (left) goal- and (right) sign-tracking conditioned responses. Mean + SEM of (A) number of food cup contacts, (B) probability of food cup contact and (C) latency to food cup contact for bLR animals (Lesion, $n=12$; Sham, $n=10$ ). For bHR animals (Lesion, $n=9$; Sham, $n=12$ ), (D) number of lever contacts, (E) probability of lever contact and (F) latency to lever contact.

bLR Sham rats during the ITIs, these differences were not as pronounced as those on measures of goal-tracking (Fig. 3A-C; Fig. S2).

Note that a small subset of bLRs did not consume all of their pellets during training, and three had to be excluded from the study for consistently leaving the majority of their pellets behind. Importantly, bLR Lesion and bLR Sham groups did not differ in their pellet consumption, and just a few rats $(n=3$ or 4$)$ from both groups left an average of 5-7 pellets behind on any given training day. The number of omissions - or trials in which no sign- or goal-tracking response occurred - was also analysed. Although rats in the bLR Lesion group had a tendency to make more omissions relative to the

bLR Sham group, this effect was not statistically significant (effect of Treatment: $F_{1,20}=3.83, P=0.06$ ). Likewise, there was not a significant Treatment $\times$ Session interaction $\left(F_{11,20}=1.78\right.$, $P=0.13$ ). Thus, taken together, we do not believe that the PVT lesions generally affected motivation to consume the food pellet or general locomotor activity in these subjects. These findings therefore demonstrate that PVT lesions selectively affect the development of a goal-tracking response in bLR rats that are inherently predisposed towards this behavior.

\section{Effects of PVT lesions in bHR rats}

For bHRs, lesions of the PVT affected the acquisition of sign-tracking behaviors (Fig. 3D-F), but only during the latter phases of training. Linear mixed effects analysis revealed a significant Session $\times$ Treatment interaction for the number of lever contacts $\left(F_{11,107}=2.04, P=0.03\right.$; Fig. 3D $)$ and the probability of lever contact $\left(F_{11,107}=3.12, P=0.01\right.$; Fig. $\left.3 \mathrm{E}\right)$. For lever contacts, there was a significant effect of Session for both bHR Lesion $\left(F_{11,107}=3.75, \quad P=0.001\right)$ and bHR Sham $\left(F_{11,107}=2.14\right.$, $P=0.02)$ groups. However, when comparing the first training session with each subsequent session, lever contacts during the latter phases of training (sessions 9-12) were significantly different from early training (session 1) only for bHR Lesion rats $(P<0.02$; Fig. 3D). For probability of lever contact, a similar pattern was evident with a significant effect of Session for both bHR Lesion $\left(F_{11,19}=7.50, P=0.001\right)$ and bHR Sham groups $\left(F_{11,19}=7.99\right.$, $P=0.001)$. The behavior of bHR Lesion rats during the latter phases of training (sessions 10-12) significantly differed from the first session $(P<0.05)$, whereas bHR Sham rats only significantly differed between session 12 and session 1 ( $P=0.03$; Fig. 3E). For latency to contact the lever (Fig. 3F), there was a significant effect of Session $\left(F_{11,115}=5.85, P=0.001\right)$, but no effect of Treatment and no Session $\times$ Treatment interaction. These data suggest that both bHR sham and bHR lesion rats learned a sign-tracking CR, as both approached the lever with decreased latency over time. Note also that there were no significant differences between sham-treated and lesion rats on goal-tracking behavior (Fig. S1A-C) or behavior during the ITI (data not shown). In sum, PVT lesions appeared to enhance sign-tracking behavior, as evident in the increased number of contacts and greater probability of contacting the lever in the latter phases of training. Although the effects of PVT lesions were less pronounced on sign-tracking behavior in bHRs compared with goaltracking behavior in bLRs, these data suggest that the PVT is involved in regulating the development of both CRs.

\section{Experiment 2: The effects of PVT lesions on the expression of sign- and goal-tracking CRs}

\section{Individual variation in PCA behavior}

Similar to previous reports (Flagel et al., 2009; Robinson \& Flagel, 2009; Meyer et al., 2012), considerable variation was seen in the form of the $\mathrm{CR}$ acquired by individual rats following seven sessions of Pavlovian training (Fig. S3). Some rats came to preferentially direct their behavior towards the lever-cue, and were classified as STs $(n=37)$. Other rats directed their behavior towards the food cup upon lever-cue presentation, indicative of a goal-tracking CR $(n=33)$. The remaining rats showed a mixed response in that they vacillated between the lever-cue and the food cup, and these rats were classified as intermediate responders $(n=50)$, and were not included in the study (data not shown). 


\section{Histology}

All subjects were screened for the presence of a PVT lesion identical to the procedures described for Experiment 1 . Figure 4 shows a schematic representation of the lesion size and location for animals included in the study. In general, lesions spanned -1.6 to $-3.4 \mathrm{~mm}$ posterior to bregma, encompassed the entire PVT, and only minimally damaged the mediodorsal, intermediodorsal or centromedial thalamic nuclei. Based on the stated criteria, 13 ST and 11 GT lesion animals were excluded from the study. One additional GT was excluded from the study because a lesion could not be verified. The final numbers of included animals were: ST Lesion $n=11$, ST Sham $n=13$, GT Lesion $n=9$, GT Sham $n=12$ (see Supporting Information and Fig. S4 for an analysis of the effects of missed lesions).

\section{Effects of PVT lesions on the expression of sign-and goal-tracking} CRs

Given that these rats were classified as sign- and goal-trackers based on their PCA Index, and that the aim of the study was to compare the effects of PVT lesions on the previously acquired CR, statistical comparisons were only made between treatment groups within a given phenotype. To assess the effects of PVT lesions on the expression of sign- and goal-tracking CRs, we assessed longitudinal changes in post-lesion behavior across multiple sessions. To do this, all measures were normalized to pre-lesion baseline levels of responding for each individual rat by subtracting the average of sessions 5-7 (i.e. baseline) from the value for each of the post-lesion sessions, 8-21. Linear mixed-effects models were then used to assess changes in behavior across sessions with the normalized value as the dependent variable. For animals that were characterized as STs, there were no significant effects of PVT lesions on measures
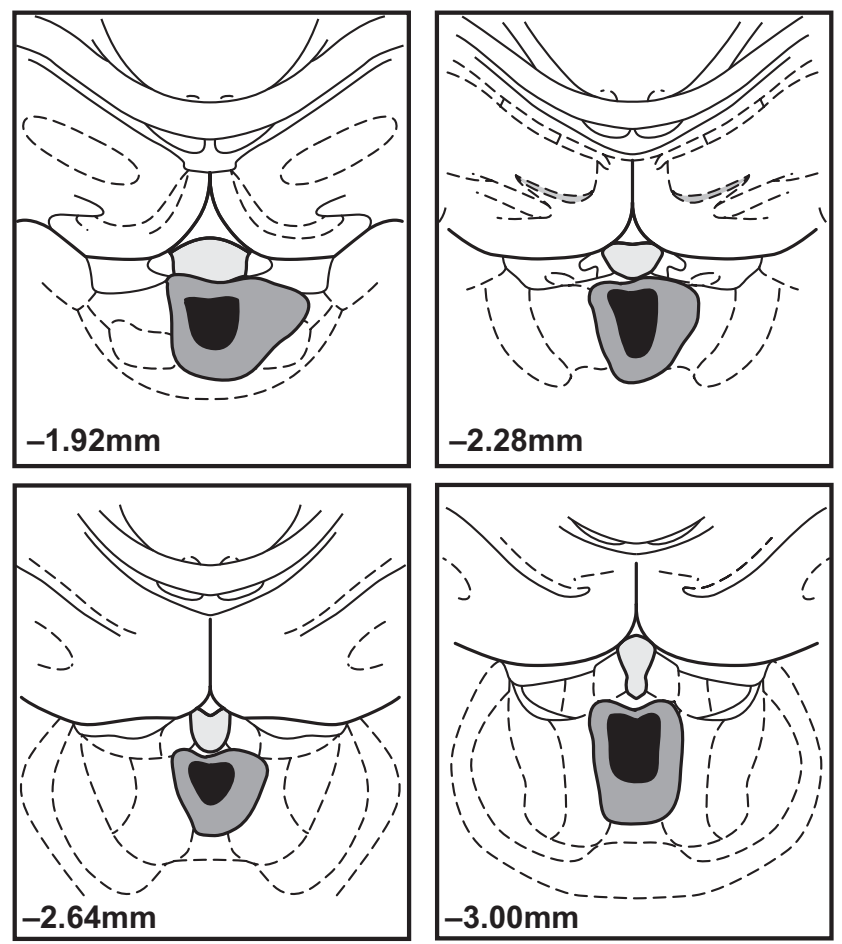

FIG. 4. Illustration showing the approximate largest (gray) and smallest (black) excitotoxic lesions of the PVT for rats included in Experiment 2. of goal- or sign-tracking behaviors or on behavior during the ITI (data not shown).

However, for rats characterized as GTs, PVT lesions after the acquisition of the CR resulted in significant changes in both goaland sign-tracking behaviors. For goal-tracking measures, there was a significant effect of Treatment for contacts with the food cup $\left(F_{13,119}=4.22, P=0.05\right.$; Fig. 5A $)$ and for latency to food cup entry $\left(F_{13,20}=4.92, P=0.04\right.$; Fig. $\left.5 \mathrm{C}\right)$, but no significant interactions on these measures. There was, however, a significant Session $\times$ Treatment interaction for probability of food cup entries $\left(F_{13,119}=2.73, P=0.02\right.$; Fig. $\left.5 \mathrm{~B}\right)$. GT Lesion rats decreased their probability of food cup entry with continued training (effect of Ses-
Goal-Tracking behavior

A

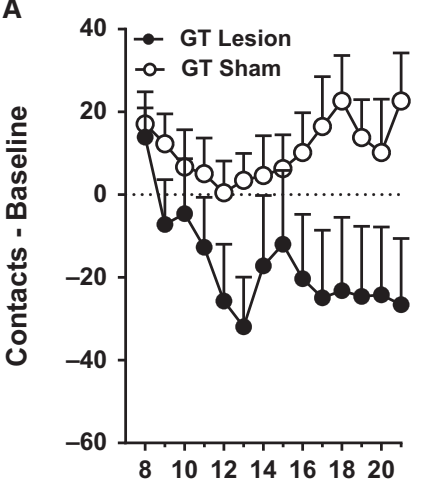

B

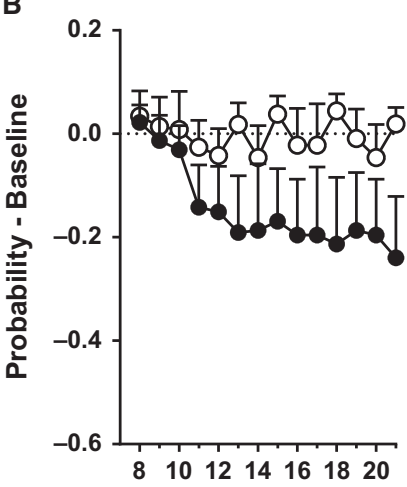

C

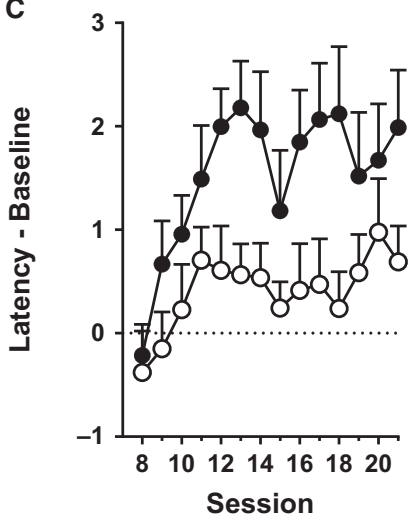

Sign-Tracking behavior

D

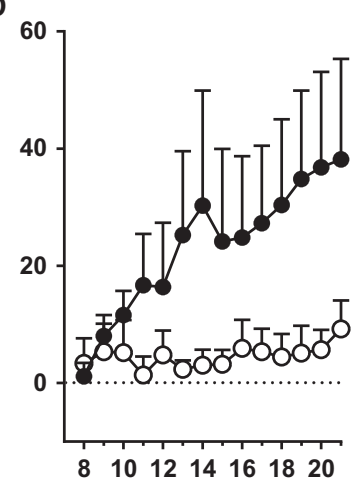

E

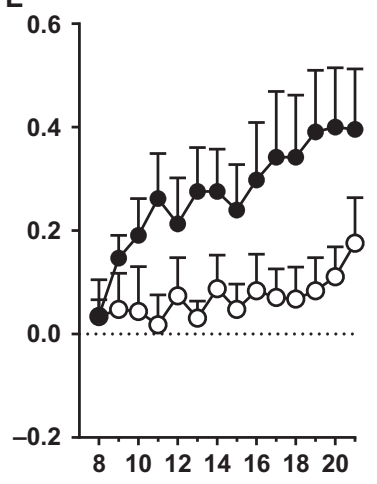

$\mathbf{F}$

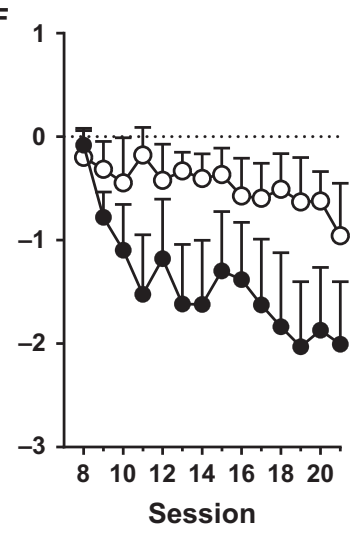

FIG. 5. GTs learn to sign-track across sessions following PVT lesions. Mean + SEM of the subtraction of pre-lesion baseline behavior (average of sessions 5-7) from post-lesion data on sessions 8-21 for (A) number of food cup contacts, (B) probability of food cup contact, (C) latency to food cup contact, (D) number of lever contacts, (E) probability of lever contact and (F) latency to lever contact (GT Lesion, $n=9$; GT Sham, $n=12$ ). 
sion for GT Lesion group; $F_{13,19}=3.91, P=0.001$ ), whereas GT Sham animals did not show a significant decrease over time. In support of this, there were significant differences between the GT Lesion and GT Sham groups later in training (i.e. sessions 15, 18 and $21 ; P<0.05)$ for probability of food cup contact. These findings demonstrate that PVT lesions attenuate the expression of goaltracking behavior in animals previously classified as GTs. Additionally, this reduction was not an immediate result of PVT lesion, but was a learned effect over the course of post-lesion training. That is, probability to approach the food magazine during the CS period diminished as a function of session. Importantly, this decrease in goal-tracking behavior is not the product of increased omissions (no effect of Treatment, $F_{1,20}=0.21, P=0.65$; no Session $\times$ Treatment interaction, $F_{20,195}=1.58, P=0.06$ ).

There were also significant differences between GT Lesion and GT Sham rats on all measures of sign-tracking behavior. There was a significant Session $\times$ Treatment interaction for lever contacts $\left(F_{13,19}=4.63, P=0.001\right.$; Fig. $\left.5 \mathrm{D}\right)$. For probability to contact the lever there was a significant effect of Treatment $\left(F_{1,19}=5.22\right.$, $P=0.03)$ and Session $\times$ Treatment interaction $\left(F_{13,19}=5.24\right.$, $P=0.001$; Fig. 5E). There was also a significant Session $\times$ Treatment interaction for latency to contact the lever $\left(F_{13,19}=2.71\right.$, $P=0.02$; Fig. 5F). Post hoc analyses show that both GT Lesion (effect of Session; $F_{13,19}=4.56, P=0.002$ ) and GT Sham (effect of Session; $F_{13,19}=2.75, P=0.02$ ) rats showed an increase in lever contacts as training progressed, but GT Lesion rats appeared to do so to a greater extent (Fig. 5D). The GT Lesion group also showed a robust increase over time for the probability to contact the lever (effect of Session; $F_{13,19}=5.12, P=0.001$ ), and there was not a significant within-group effect of Session for the GT Sham group. A similar pattern was evident for the latency to contact the lever, with the GT Lesion group showing decreased latency (effect of Session; $\left.F_{13,19}=2.52, P=0.03\right)$ to approach the lever over time, whereas sham controls exhibited relatively stable behavior on this measure. These results demonstrate that, for rats previously characterized as GTs, PVT lesions resulted in a significant increase in sign-tracking behavior. Furthermore, for all measures, the GT Lesion and GT
Sham groups significantly differed no sooner than session 11, again indicating that the increase in lever-directed behavior in the GT Lesion group was not an immediate effect of the PVT lesion, but was a product of post-lesion Pavlovian training.

To examine whether PVT lesions had changed animals previously classified as GTs into STs, a repeated-measures ANOVA was used to compare pre-lesion (pre-lesion block; average of sessions 5-7) with post-lesion (post-lesion block; average of sessions 19-21) changes in response bias score (Fig. 6A). Response bias is an index of an individual's bias towards the lever-cue vs. the food magazine during CS presentation, which is calculated using the following formula: [(total lever-directed contacts) - (total food cup-directed contacts) $] \div$ (sum of total contacts). As a result, scores close to 1.0 represent behavior directed exclusively toward the lever (sign-tracking), while scores near -1.0 represent behavior directed exclusively towards the food cup (goal-tracking). Repeated-measures ANOvA showed a significant Block $\times$ Treatment interaction $\left(F_{1,19}=6.02, P=0.02\right)$ for response bias score. Post-hoc analyses indicated a significant change in prevs. post-lesion behavior $(P<0.001)$ for the GT Lesion group, but not the GT Sham control group. In agreement with this, there was a significant difference between GT Lesion and GT Sham groups for the post-lesion block $(P=0.05)$. Prior to surgery, both GT Lesion and GT Sham groups showed response bias scores around -0.9 , indicating behavior was primarily directed towards the food cup. Following surgery, however, the response bias score of the lesion group moved to approximately -0.15 , while the sham group stayed stable at approximately -0.75 . A response bias score of -0.15 indicates that GTs with PVT lesions are not pure STs, but are showing an intermediate phenotype. Thus, their behavior is vacillating between the lever and the food cup; or, on a given trial, a rat may exhibit aspects of both a sign- and a goal-tracking response (e.g. Fig. 6B).

\section{Discussion}

Here we assessed the effects of an excitotoxic lesion of the PVT on the acquisition and expression of sign- and goal-tracking CRs. Our results indicate that the PVT is required for the acquisition of a

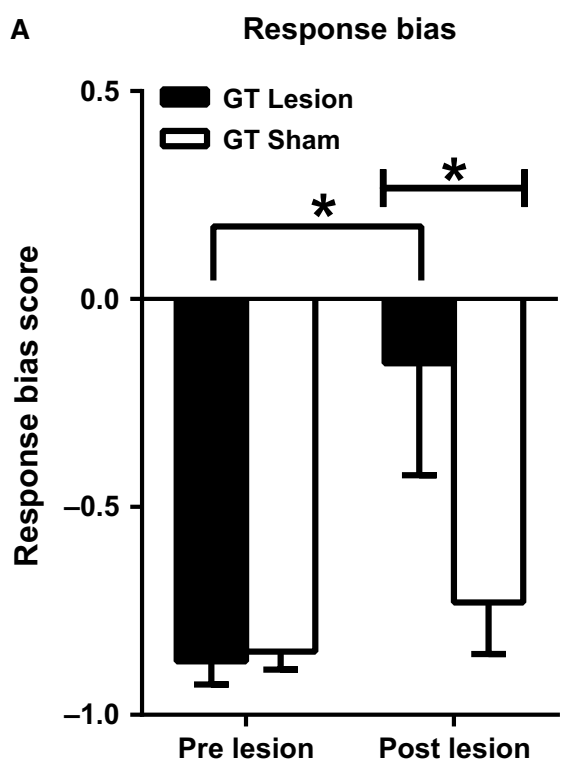

B

Intermediate response

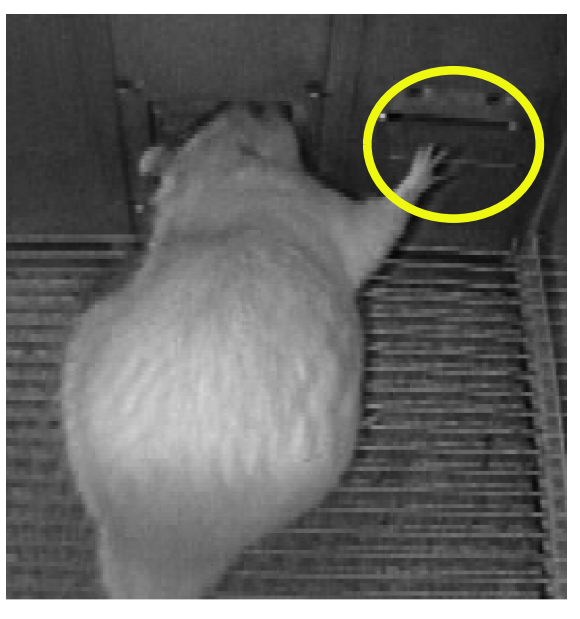

FIG. 6. PVT lesions increase the tendency to sign-track in GTs. Mean + SEM of (A) pre-lesion baseline behavior (average of sessions 5-7) and post-lesion behavior (average of sessions 19-21) for response bias score [(lever contacts - food cup contacts) $\div$ (total contacts)]. The GT Lesion group $(n=9)$ showed a significant increase in response bias score following PVT lesion $(P<0.001)$. The GT Lesion group also differed significantly from the sham control group $(n=12)$ post-lesion $(P=0.05)$. (B) A video image showing a rat performing an 'intermediate' response. 
goal-tracking CR, but not a sign-tracking CR. In fact, lesioning the PVT prior to Pavlovian learning results in an exaggerated sign-tracking $\mathrm{CR}$ later in training. In addition, lesioning the PVT following the acquisition of sign- and goal-tracking CRs does not affect the behavior of STs, but leads to an overall shift towards sign-tracking behavior in animals previously classified as GTs. These data suggest that the PVT is involved in mediating both sign- and goal-tracking behaviors, but in different ways.

A great advantage of utilizing the bHR/bLR rat lines is the ability to know a priori what CR the animals will develop, which was critical for examining the effects of PVT lesions on the acquisition of sign- and goal-tracking behaviors. Interestingly, locomotor response to novelty and the propensity to attribute incentive salience to reward cues (i.e. sign-tracking) are not highly correlated in outbred rats, as they are in the bred lines (Flagel et al., 2010). In fact, the bHR/bLR rats differ on several genetic, neurobiological and behavioral traits that do not normally segregate with one another in outbred populations (for a detailed review, see Flagel et al., 2014). Thus, any number of factors inherent to the bred lines could affect their initial tendency to sign- or goal-track, as well as any subsequent effects of manipulations, such as the lesion effects shown here. Nonetheless, any concerns due to the use of the selectively bred rats in the Acquisition Experiment are mitigated by the fact that comparable results were found when commercially available outbred rats were used for the Expression Experiment. Furthermore, additional analyses ruled out potential 'general' effects of locomotor activity or motivational drive to obtain food in these studies, reinforcing the fact that findings from the Acquisition Experiment utilizing the bHR/bLR rats are indeed due to lesion effects on PCA behavior.

In the Acquisition Experiment we found that the bHR Sham rats developed a sign-tracking CR consistent with previous findings (Flagel et al., 2010, 2011b). The bLR Sham rats, however, did not develop as robust a goal-tracking response as that seen in previous studies (Flagel et al., 2010, 2011b). It is likely that the attenuated goal-tracking response in bLR rats in this study was due to extremely low levels of locomotor activity that have become characteristic of recent generations of bLR rats. Nonetheless, this does not detract from the current findings. In fact, had the bLR Sham group shown a more prominent goal-tracking response, the differences between the sham and lesioned animals would have been more pronounced. It is also important to note that there were no significant differences in trial omissions between bLR Lesion and Sham groups, and nor were there any differences in the number of reward pellets consumed. Thus, we believe the reduction in goal-tracking behavior seen in the bLR Lesion group is specific to the CR, and not due to a general loss of motivation or locomotor function in these animals.

Interestingly, when PVT lesions were performed following the acquisition of the $\mathrm{CR}$ in commercially available outbred rats, there were no effects on either sign- or goal-tracking behavior in rats characterized as STs. This was surprising because, in selectively bred HR rats, PVT lesions prior to CR acquisition led to increased asymptotic performance of a sign-tracking CR. Note, however, that the levels of sign-tracking behavior exhibited by the outbred rats (Fig. S3D-F) were greater than those exhibited by the bHR Sham rats (Fig. 3D-F). Thus, the most likely explanation for these seemingly discrepant results is that PVT lesions could not further enhance the performance of STs in the Expression Experiment because of a ceiling effect. To test this hypothesis, future studies might consider using rats that are intermediate responders to see if a PVT lesion could render these animals STs.

Perhaps the most interesting findings presented here are the effects of PVT lesions after outbred rats had acquired a goal-tracking
CR. GTs with PVT lesions exhibited an attenuated goal-tracking CR relative to sham controls, which was consistent with the findings from the Acquisition Experiment using bLR rats. Concomitant with this reduction in goal-tracking behavior, however, was an increase in lever-directed behaviors, or sign-tracking. This behavioral shift was not apparent immediately following the PVT lesion, but developed over the course of post-lesion training. As a result, GT Lesion rats shifted to an intermediate phenotype by the end of training. That is, goal-tracking rats with a PVT lesion began to show increased interest in the lever upon its presentation, vacillating between it and the food cup. This is especially interesting as it is the first evidence to show that animals expressing a goal-tracking $\mathrm{CR}$ can be biased towards a sign-tracking $\mathrm{CR}$ via a neurobiological manipulation.

The fact that the behavioral shift towards sign-tracking for rats previously characterized as GTs was not immediately apparent following the PVT lesion argues against the possibility that this effect could be attributed to an overall increase in locomotor activity. In agreement, others have assessed locomotor behavior following PVT lesions, and have found no differences between lesion and sham groups (Pierce et al., 1997; Young \& Deutch, 1998; Hamlin et al., 2009). Furthermore, we did not see an effect of PVT lesions when we assessed locomotor response to novelty in a subset of outbred animals from the Expression Experiment (Fig. S5; for detailed Materials and Methods and Results, see Supporting Information). Thus, we do not believe the increase in sign-tracking behavior reported here was due to an increase in general locomotor behavior.

In light of these results, it is interesting that an increased tendency to develop a sign-tracking CR following PVT lesion was not apparent in the bLR Lesion group in the Acquisition Experiment. However, as discussed above, this could be due to the overall lack of activity that is characteristic of the bLR lines. Thus, if the Acquisition Experiment was repeated with a population of outbred rats, it is possible that one would observe an overall population shift towards sign-tracking in PVT lesion animals. Unfortunately, it is difficult to interpret acquisition studies in outbred populations because it is impossible to know what $\mathrm{CR}$ rats will develop prior to training.

Another point to consider upon interpretation of the Expression Experiment is that learning might have still been occurring at the time of the lesion. Although numerous studies have shown that rats acquire sign- and goal-tracking CRs within the first week of training (Flagel et al., 2009, 2011a,b; Robinson \& Flagel, 2009; Meyer et al., 2012), we cannot rule out the possibility that ongoing learning processes are present even after rats have begun to exhibit their respective CRs. As shown in Fig. S2, however, both STs and GTs appear to have reached stable levels of responding by session 7 , prior to the lesion. Thus, the apparent effects are probably specific to the ongoing performance of the CRs, rather than the learning process per se.

The current study was largely driven by previous findings showing that, relative to GTs, STs exhibit enhanced c-fos expression in the PVT in response to food- and drug-paired cues (Flagel et al., 2011a; Yager et al., 2014). Thus, it appears that only if a rewardcue is attributed with incentive salience will it robustly activate the PVT. In addition, correlations of cue-induced c-fos levels across brain regions within STs and GTs reveal different patterns of 'functional connectivity' (Flagel et al., 2011a; Haight \& Flagel, 2014). Interestingly, one of the main points of divergence with this analysis was the PVT. For STs, cue-induced c-fos mRNA in the PVT was correlated with that in the shell of the nucleus accumbens (NAc), whereas GTs showed correlated levels of c-fos mRNA between the PVT and areas of the prefrontal cortex, particularly the prelimbic 
cortex (PrL). This suggests that, in response to cue presentation, STs and GTs might be utilizing different neural circuitry that converges on the PVT.

Our current data expand upon these recent findings, and affirm an important role for the PVT in both sign- and goal-tracking behavior. The findings stated above, namely correlated cue-induced c-fos mRNA between the PrL and PVT in GTs (Haight \& Flagel, 2014), is consistent with the fact that the PVT receives dense cortical input from layer six of the PrL (Li \& Kirouac, 2012). The PrL is known to be critical for goal-directed behavior (Balleine \& Dickinson, 1998), but its role in these behaviors is complicated and not fully understood. For instance, inactivation of the PrL can facilitate or inhibit reinstatement of alcohol seeking in rats, depending on the context in which this behavior was extinguished (Willcocks \& McNally, 2013). One view is that the PrL acts as a locus of 'cognitive-control', capable of inhibiting responses to reward-paired cues (Jonkman et al., 2009; Kober et al., 2010; Mihindou et al., 2013). In this regard, it is possible that STs and GTs differ in their degree of 'cognitive control' of their behavior, and PrL afferents to the PVT may be a critical component of this top-down circuitry. Specifically, these afferents might serve to suppress the motivational drive from subcortical areas, such as the hypothalamus, that are activated by presentation of reward-paired cues (Choi et al., 2010; Mahler et al., 2012). In the current study, it is therefore plausible that lesions of the PVT result in a loss of inhibitory control, presumably releasing the 'brake' on sign-tracking behavior. Thus, following PVT lesions, individuals who were previously GTs appear to relearn the cue-reward association using a different strategy - one that allows the cue to become attributed with incentive salience.

While these top-down cognitive processes might be stronger in GTs, it is also possible that sub-cortical motivational circuitry could be overriding this cortical control in STs. The PVT also receives input from subcortical brain areas known to be involved in motivated behavior, including dopamine and orexin projections from the hypothalamus (Kirouac et al., 2005; Parsons et al., 2006; Li et al., 2014). Inside the PVT, these sub-cortical motivational signals are probably integrated with cognitive control signals from the PrL, to ultimately influence the activity of PVT neurons that project to the NAc. Importantly, these PVT efferents can modulate NAc dopamine release (Jones et al., 1989; Parsons et al., 2007), which is critical for sign-tracking behavior (Flagel et al., 2011b; Saunders \& Robinson, 2012). However, these signals would need to be strong enough to override the top-down control coming from the PrL to the PVT. Therefore, if the sub-cortical afferents to the PVT were specifically inhibited while leaving the PrL afferents intact, it is likely that one would see an attenuation of sign-tracking behavior. Although this is not what we report in the current study, future studies will incorporate techniques capable of systematically targeting different components of this circuitry to determine what is driving sign-tracking behavior.

It has recently been shown that contextual, and not discrete, reward-paired cues can preferentially acquire motivational control over behavior in GTs compared with STs (Saunders et al., 2014). Interestingly, previous reports have shown that exposure to contextual cues previously associated with administration of a highly palatable food (Schiltz et al., 2005a, 2007), nicotine (Schiltz et al., 2005b) or cocaine (Johnson et al., 2010) can elicit robust immediate early gene expression in the PVT, including c-fos expression. In addition, lesions or chemical inactivation of the PVT can attenuate contextual cue-induced reinstatement of alcohol-seeking behavior (Hamlin et al., 2009; Marchant et al., 2010), and the expression of cocaine-induced conditioned place preference is attenuated following inactivation of the PVT with GABA receptor agonists (Browning et al., 2014). On a neuroanatomical level, these data are congruent with the fact that the PVT receives input from the ventral subiculum of the hippocampus (Li \& Kirouac, 2012), an area critical for context-induced reinstatement of drug-seeking behavior (Sun \& Rebec, 2003; Lasseter et al., 2010). In addition, prelimbic afferents to the PVT might also be playing a role, as PrL function is needed to use contextual cues to guide goal-directed behavior (Marquis et al., 2007). Given these recent findings (Hamlin et al., 2009; Marchant et al., 2010; Browning et al., 2014; Saunders et al., 2014), we postulate that the PVT might also be critically involved in the attribution of incentive motivational values to contextual cues. Thus, the attenuation of the goal-tracking response in lesioned GTs could have been due to a loss of motivational significance from contextual stimuli, resulting in behavior biased more towards the discrete cue (i.e. sign-tracking). Future studies will further assess the role of ventral subiculum and PrL afferents to the PVT, and whether these pathways play an important role in mediating the motivational significance of contextual cues.

In conclusion, we used an animal model that captures individual variation in response to discrete reward cues to assess the role of the PVT in different cue-reward learning processes. Using an excitotoxic lesion, we showed that PVT lesions attenuate the development of a goal-tracking CR, while increasing a sign-tracking CR. Taken together, these data support a role for the PVT in regulating individual differences in conditioned responding to discrete Pavlovian-conditioned reward cues. Specifically, the PVT may serve as a key node that regulates the attribution of incentive motivational values to reward-paired cues. Ongoing studies will further dissect the role of specific PVT efferents and afferents in incentive salience attribution to reward-paired cues.

\section{Supporting Information}

Additional supporting information can be found in the online version of this article:

Table S1. Statistical results for Experiment 1.

Fig. S1. Effects of PVT lesion on the acquisition of 'off target' behaviors in bHR and bLR animals.

Fig. S2. Activity during the inter-trial interval for bLR rats.

Fig. S3. The acquisition of sign- and goal-tracking conditioned responses across seven PCA training sessions.

Fig. S4. The extent of PVT lesion underlies change in response bias score.

Fig. S5. PVT lesions do not affect locomotor response to a novel environment.

\section{Acknowledgements}

Funding for this work was provided by the National Institute on Drug Abuse predoctoral training grant T32 DA007821 (J.L.H.) and individual National Research Service Award F31 DA037680 (J.L.H.), as well as The Office of Naval Research grant ONR:N00014-02-1-0879 (H.A.) and internal funds from the Department of Psychiatry at the University of Michigan (S.B.F.). The authors have no conflicts of interest to disclose. The authors thank Katie Long for her assistance with the behavioral portion of the experiments, as well as Peter Blandino, Angela Koelsch and James Stewart for their assistance with the bHR/bLR breeding colony. In addition, we thank Dr Terry Robinson for comments on an earlier version of this manuscript.

\section{Abbreviations}

bHR, bred high-responder; bLR, bred low-responder; CM, central medial thalamic nucleus; CR, conditioned response; CS, conditioned stimulus; GT, 
goal-tracker; ITI, inter-trial interval; NAc, nucleus accumbens; PCA, Pavlovian conditioned approach; PrL, prelimbic cortex; PVT, paraventricular nucleus of the thalamus; ST, sign-tracker.

\section{References}

Balleine, B.W. \& Dickinson, A. (1998) Goal-directed instrumental action: contingency and incentive learning and their cortical substrates. Neuropharmacology, 37, 407-419.

Browning, J.R., Jansen, H.T. \& Sorg, B.A. (2014) Inactivation of the paraventricular thalamus abolishes the expression of cocaine conditioned place preference in rats. Drug Alcohol Depen., 134, 387-390.

Choi, D.L., Davis, J.F., Fitzgerald, M.E. \& Benoit, S.C. (2010) The role of orexin-A in food motivation, reward-based feeding behavior and food-induced neuronal activation in rats. Neuroscience, 167, 11-20.

Dayas, C.V., McGranahan, T.M., Martin-Fardon, R. \& Weiss, F. (2008) Stimuli linked to ethanol availability activate hypothalamic CART and orexin neurons in a reinstatement model of relapse. Biol. Psychiat., 63, 152-157.

Fitzpatrick, C.J., Gopalakrishnan, S., Cogan, E.S., Yager, L.M., Meyer, P.J., Lovic, V., Saunders, B.T., Parker, C.C., Gonzales, N.M., Aryee, E., Flagel, S.B., Palmer, A.A., Robinson, T.E. \& Morrow, J.D. (2013) Variation in the form of Pavlovian conditioned approach behavior among outbred male Sprague-Dawley rats from different vendors and colonies: sign-tracking vs. goal-tracking. PLoS One, 8, e75042.

Flagel, S.B., Watson, S.J., Robinson, T.E. \& Akil, H. (2007) Individual differences in the propensity to approach signals vs goals promote different adaptations in the dopamine system of rats. Psychopharmacology, 191, 599-607.

Flagel, S.B., Watson, S.J., Akil, H. \& Robinson, T.E. (2008) Individual differences in the attribution of incentive salience to a reward-related cue: influence on cocaine sensitization. Behav. Brain Res., 186, 48-56.

Flagel, S.B., Akil, H. \& Robinson, T.E. (2009) Individual differences in the attribution of incentive salience to reward-related cues: implications for addiction. Neuropharmacology, 56(Suppl 1), 139-148.

Flagel, S.B., Robinson, T.E., Clark, J.J., Clinton, S.M., Watson, S.J., Seeman, P., Phillips, P.E. \& Akil, H. (2010) An animal model of genetic vulnerability to behavioral disinhibition and responsiveness to reward-related cues: implications for addiction. Neuropsychopharmacology, 35, 388-400.

Flagel, S.B., Cameron, C.M., Pickup, K.N., Watson, S.J., Akil, H. \& Robinson, T.E. (2011a) A food predictive cue must be attributed with incentive salience for it to induce c-fos mRNA expression in cortico-striatal-thalamic brain regions. Neuroscience, 196, 80-96.

Flagel, S.B., Clark, J.J., Robinson, T.E., Mayo, L., Czuj, A., Willuhn, I., Akers, C.A., Clinton, S.M., Phillips, P.E. \& Akil, H. (2011b) A selective role for dopamine in stimulus-reward learning. Nature, 469, 53-57.

Flagel, S.B., Waselus, M., Clinton, S.M., Watson, S.J. \& Akil, H. (2014) Antecedents and consequences of drug abuse in rats selectively bred for high and low response to novelty. Neuropharmacology, 76(Part B), 425-436.

Haight, J.L. \& Flagel, S.B. (2014) A potential role for the paraventricular nucleus of the thalamus in mediating individual variation in Pavlovian conditioned responses. Front. Behav. Neurosci., 8, 79.

Hamlin, A.S., Clemens, K.J., Choi, E.A. \& McNally, G.P. (2009) Paraventricular thalamus mediates context-induced reinstatement (renewal) of extinguished reward seeking. Eur. J. Neurosci., 29, 802-812.

Igelstrom, K.M., Herbison, A.E. \& Hyland, B.I. (2010) Enhanced c-Fos expression in superior colliculus, paraventricular thalamus and septum during learning of cue-reward association. Neuroscience, 168, 706-714.

James, M.H. \& Dayas, C.V. (2013) What about me...? The PVT: a role for the paraventricular thalamus (PVT) in drug-seeking behaviour. Front. Behav. Neurosci., 7, 18.

James, M.H., Charnley, J.L., Flynn, J.R., Smith, D.W. \& Dayas, C.V. (2011) Propensity to 'relapse' following exposure to cocaine cues is associated with the recruitment of specific thalamic and epithalamic nuclei. Neuroscience, 199, 235-242.

Johnson, Z.V., Revis, A.A., Burdick, M.A. \& Rhodes, J.S. (2010) A similar pattern of neuronal Fos activation in 10 brain regions following exposure to reward- or aversion-associated contextual cues in mice. Physiol. Behav., 99, 412-418.

Jones, M.W., Kilpatrick, I.C. \& Phillipson, O.T. (1989) Regulation of dopamine function in the nucleus accumbens of the rat by the thalamic paraventricular nucleus and adjacent midline nuclei. Exp. Brain Res., 76, $572-580$.

Jonkman, S., Mar, A.C., Dickinson, A., Robbins, T.W. \& Everitt, B.J. (2009) The rat prelimbic cortex mediates inhibitory response control but not the consolidation of instrumental learning. Behav. Neurosci., 123, $875-885$.

Kelley, A.E., Baldo, B.A. \& Pratt, W.E. (2005) A proposed hypothalamicthalamic-striatal axis for the integration of energy balance, arousal, and food reward. J. Comp. Neurol., 493, 72-85.

Kirouac, G.J., Parsons, M.P. \& Li, S. (2005) Orexin (hypocretin) innervation of the paraventricular nucleus of the thalamus. Brain Res., 1059, 179-188.

Kober, H., Mende-Siedlecki, P., Kross, E.F., Weber, J., Mischel, W., Hart, C.L. \& Ochsner, K.N. (2010) Prefrontal-striatal pathway underlies cognitive regulation of craving. Proc. Natl. Acad. Sci. USA, 107, 14811-14816.

Lasseter, H.C., Xie, X., Ramirez, D.R. \& Fuchs, R.A. (2010) Sub-region specific contribution of the ventral hippocampus to drug context-induced reinstatement of cocaine-seeking behavior in rats. Neuroscience, 171, 830 839.

Li, S. \& Kirouac, G.J. (2012) Sources of inputs to the anterior and posterior aspects of the paraventricular nucleus of the thalamus. Brain Struct. Funct., 217, 257-273.

Li, S., Shi, Y. \& Kirouac, G.J. (2014) The hypothalamus and periaqueductal gray are the sources of dopamine fibers in the paraventricular nucleus of the thalamus in the rat. Front. Neuroanat., 8, 136.

Mahler, S.V., Smith, R.J., Moorman, D.E., Sartor, G.C. \& Aston-Jones, G. (2012) Multiple roles for orexin/hypocretin in addiction. Prog. Brain Res., 198, 79-121.

Marchant, N.J., Furlong, T.M. \& McNally, G.P. (2010) Medial dorsal hypothalamus mediates the inhibition of reward seeking after extinction. $J$. Neurosci., 30, 14102-14115.

Marquis, J.-P., Killcross, S. \& Haddon, J.E. (2007) Inactivation of the prelimbic, but not infralimbic, prefrontal cortex impairs the contextual control of response conflict in rats. Eur. J. Neurosci., 25, 559-566.

Martin-Fardon, R. \& Boutrel, B. (2012) Orexin/hypocretin (Orx/Hcrt) transmission and drug-seeking behavior: is the paraventricular nucleus of the thalamus (PVT) part of the drug seeking circuitry? Front. Behav. Neurosci., 6, 75 .

Meyer, P.J., Lovic, V., Saunders, B.T., Yager, L.M., Flagel, S.B., Morrow, J.D. \& Robinson, T.E. (2012) Quantifying individual variation in the propensity to attribute incentive salience to reward cues. PLoS One, 7, e38987.

Mihindou, C., Guillem, K., Navailles, S., Vouillac, C. \& Ahmed, S.H. (2013) Discriminative inhibitory control of cocaine seeking involves the prelimbic prefrontal cortex. Biol. Psychiat., 73, 271-279.

Parsons, M.P., Li, S. \& Kirouac, G.J. (2006) The paraventricular nucleus of the thalamus as an interface between the orexin and CART peptides and the shell of the nucleus accumbens. Synapse, 59, 480-490.

Parsons, M.P., Li, S. \& Kirouac, G.J. (2007) Functional and anatomical connection between the paraventricular nucleus of the thalamus and dopamine fibers of the nucleus accumbens. J. Comp. Neurol., 500, 1050-1063.

Pierce, R.C. \& Kalivas, P.W. (1997) A circuitry model of the expression of behavioral sensitization to amphetamine-like psychostimulants. Brain Res. Rev., 25, 192-216.

Pierce, R.C., Reeder, D.C., Hicks, J., Morgan, Z.R. \& Kalivas, P.W. (1997) Ibotenic acid lesions of the dorsal prefrontal cortex disrupt the expression of behavioral sensitization to cocaine. Neuroscience, 82, 1103-1114.

Robinson, T.E. \& Flagel, S.B. (2009) Dissociating the predictive and incentive motivational properties of reward-related cues through the study of individual differences. Biol. Psychiat., 65, 869-873.

Saunders, B.T. \& Robinson, T.E. (2012) The role of dopamine in the accumbens core in the expression of Pavlovian-conditioned responses. Eur. J. Neurosci., 36, 2521-2532.

Saunders, B.T., O’Donnell, E.G., Aurbach, E.L. \& Robinson, T.E. (2014) A cocaine context renews drug seeking preferentially in a subset of individuals. Neuropsychopharmacology, 39, 2816-2823.

Schiltz, C., Kelley, A. \& Landry, C. (2005a) Exposure to cues associated with palatable food increases immediate-early gene (IEG) mRNA and proenkephalin (PENK) premRNA expression in the rat brain. Neuropsychopharmacology, 30, S213.

Schiltz, C.A., Kelley, A.E. \& Landry, C.F. (2005b) Contextual cues associated with nicotine administration increase arc mRNA expression in corticolimbic areas of the rat brain. Eur. J. Neurosci., 21, 1703-1711.

Schiltz, C., Bremer, Q., Landry, C. \& Kelley, A. (2007) Food-associated cues alter forebrain functional connectivity as assessed with immediate early gene and proenkephalin expression. BMC Biol., 5, 16.

Stead, J.H., Clinton, S., Neal, C., Schneider, J., Jama, A., Miller, S., Vazquez, D., Watson, S. \& Akil, H. (2006) Selective breeding for divergence in novelty-seeking traits: heritability and enrichment in spontaneous anxiety-related behaviors. Behav. Genet., 36, 697-712. 
Sun, W. \& Rebec, G.V. (2003) Lidocaine inactivation of ventral subiculum attenuates cocaine-seeking behavior in rats. J. Neurosci., 23, 1025810264.

Verbeke, G. \& Molenberghs, G. (2000) Linear Mixed Models for Longitudinal Data. Springer, New York.

Vertes, R.P. \& Hoover, W.B. (2008) Projections of the paraventricular and paratenial nuclei of the dorsal midline thalamus in the rat. J. Comp. Neurol., 508, 212-237.

Wedzony, K., Koros, E., Czyrak, A., Chocyk, A., Czepiel, K., Fijal, K., Mackowiak, M., Rogowski, A., Kostowski, W. \& Bienkowski, P. (2003) Different pattern of brain c-Fos expression following re-exposure to ethanol or sucrose self-administration environment. N.-S. Arch. Pharmacol., 368, 331-341.

Willcocks, A.L. \& McNally, G.P. (2013) The role of medial prefrontal cortex in extinction and reinstatement of alcohol-seeking in rats. Eur. J. Neurosci., 37, 259-268.

Yager, L.M., Pitchers, K.K., Flagel, S.B. \& Robinson, T.E. (2014) Individual variation in the motivational and neurobiological effects of an opioid cue. Neuropsychopharmacology, 40, 1269-1277.

Young, C.D. \& Deutch, A.Y. (1998) The effects of thalamic paraventricular nucleus lesions on cocaine-induced locomotor activity and sensitization. Pharmacol. Biochem. Be., 60, 753-758. 